\title{
Body Ownership of Virtual Avatars: An Affordance Approach of Telepresence
}

\author{
Tiago Coelho ${ }^{1}$, Rita de Oliveira ${ }^{2}$, Tiago Cardoso ${ }^{1}$, and Yves Rybarczyk ${ }^{1, *}$ \\ ${ }^{1}$ Electrotechnical Engineering Department, New University of Lisbon, Portugal \\ $\{y r$, tcoelho, tomfc $\}$ auninova.pt \\ ${ }^{2}$ Department of Applied Sciences, London South Bank University, UK \\ r.oliveiralisbu.ac.uk
}

\begin{abstract}
Virtual environments are an increasing trend in today's society. In this scope, the avatar is the representation of the user in the virtual world. However, that relationship lacks empirical studies regarding the nature of the interaction between avatars and human beings. For that purpose it was studied how the avatar's modeled morphology and dynamics affect its control by the user. An experiment was conducted to measure telepresence and ownership on participants who used a Kinect Natural User Interface (NUI). The body ownership of different avatars was assessed through a behavioral parameter, based on the concept of affordances, and a questionnaire of presence. The results show that the feelings of telepresence and ownership seem to be greater when the kinematics and the avatar's proportions are closer to those of the user.
\end{abstract}

Keywords: Avatar, telepresence, ownership, affordances, natural user interface, virtual environment.

\section{Introduction}

\subsection{Telepresence and Ownership}

Telepresence is the feeling of being present in a place where the person is not [1]. This feeling can be achieved while an individual is using a simulator or performing a certain task in a virtual environment such as a game [2]. Another way this phenomenon can occur is in teleoperation, in which the user remote-controls a robot with a camera that provides the teleoperator with visual feedback from the working space [3]. Telepresence is a critical mental process as it increases the immersion of the individual within a certain task. Teleoperation and virtual environments are the most common situations in which a feeling of telepresence may occur.

One of the most specific cases of telepresence is body ownership, in which the individual is so immerse in the teleoperation task he/she is performing that he/she

\footnotetext{
* Departamento de Engenharia Electrotécnica, Faculdade de Ciências e Tecnologia, Universidade Nova de Lisboa, Quinta da Torre 2829-516 Monte de Caparica, Portugal email: yr@uninova.pt
} 
believes the remote machine is part of him/her [4]. In an experiment carried out by Sumioka et al. [5], subjects remote-controlled a human-like machine. They had a first person view over the machine, which replicated every move of the individual. The participant's reactions were gauged by measurement of the skin conductance. The results show that the participants seemed to feel the machine as it was their own body. In addition, the feeling of ownership can also happen in other cases of mediated situations such as in virtual reality, in which the individual believes he/she is the avatar.

The study that revealed for the first time the phenomenon of ownership is the Rubber Hand Illusion [6]. In this experiment, the participant's hand is hidden and a rubber hand is visible in its place. A tactile stimulation is applied simultaneously to both hands. After a while, the individual has the feeling that the fake hand is his/her own. A similar effect has also been observed in virtual reality [7, 8]. Ehrsson et al. [9] recorded the brain activity of participants when they were submitted to the same experiment. The results showed a significant activation of the parietal cortex only in the presence of a synchronous and congruent visuo-tactile stimulation of the rubber and the real hand. In addition, a positive correlation between the physiological and ownership questionnaire data confirmed that the participants were considering the rubber hand as their own hand.

It seems that the ownership feeling is not exclusive of the individual limbs and can occur on the entire body. In Petkova and Ehrsson's [10] experiment, participants wore a head-mounted display and had a first person view over a body-sized mannequin. They received visual and tactile stimulations over the whole body. In that condition, the participants had the feeling that the mannequin's body was their own. The ownership feeling was measured through skin conductance, which can detect psychological or physiological alterations. Authors stress the fact that a human-like representation of the mannequin and a synchronous visuo-tactile stimulation were crucial to trigger the ownership illusion.

A more surprising observation is the fact that body ownership may also occur in a simple situation of tool-use. Studies showed that when individuals manipulate an artifact, they consider it as an extension of their arm [11]. The initial study was performed with non-human primates and their brain activity was measured by electrodes. The results show that some specific bimodal neurons coding for the monkey's hand fire in a similar manner when a stimulus is applied to the hand or close to the tool manipulated by the animal [12]. This is clear evidence showing that the artifact seems to be integrated into the primate body schema.

Overall, these experiments showed that ownership occurs in the brain, after integration of multimodal information (vision, touch and proprioception) in order to build a coherent representation of the body.

\subsection{Methods for Measuring Telepresence}

Questionnaires. There are several ways to measure telepresence. One way the evaluation can be done is by questionnaire, in which the user answers a few questions in order to express what they felt during the experiment. This is probably the most popular kind of presence assessment. A significant number of studies on telepresence or 
ownership ask their participants to fill a questionnaire when the experiment is over $[6,9,10,13]$.

Questionnaires are mostly used because of the simplicity of their implementation and the large range of possible questions. They are also a very quick and practical way for people to express their feelings, on a numerical scale that allows quantification and comparisons. Nevertheless there are some disadvantages, such as misinterpretation of questions, subjectivity of answers, the scale level number (odd vs. even) or, since it happens after the experiment, participants might forget what they felt. Another disadvantage is the number of questions: if there are too many, the participants may lose interest and answer randomly.

Physiological Parameters. Another way to gauge presence is by physiological parameters such as heart rate, galvanic skin response, electromyography or electroencephalogram. The galvanic skin response (GSR) measures the skin conductance of electricity. The variation of skin conductance occurs by changes in the moisture of the skin. Emotional stimulus triggers the sympathetic nervous system to increase the activity of the sweat glands. Armel and Ramachandran [14] measured the skin conductance in their rubber hand illusion experiment. They threatened to harm the rubber hand. If the participant thought that the rubber hand was his/her own, the skin conductance results showed signs of arousal.

The electromyography (EMG) measures the electric activity of activated muscles. The signals can also be used to detect neurologic activity. Slater et al. [15] used this technique during a rubber hand illusion experiment carried out in virtual reality. When ownership was achieved, the virtual hand was twisted. The EMG showed the twist induced a motor activity along the participant's real arm.

The electroencephalogram (EEG) directly measures electric activity of the brain in a non-invasive way. González-Franco et al. [16] showed that EEG can be used to assess presence in a virtual environment. They conducted an experiment in which the arm of the avatar was threatened. Results showed that when facing a threat, the participants lowered their motor cortex activity.

Behavioral Assessment: Affordances. In this project, affordances are used as a behavioral assessment to measure telepresence in a virtual environment. Affordances are a concept first suggested in the literature by J.J. Gibson [17]. An affordance is an action possibility whereby people perceive their environment and the objects within it as possibilities of doing certain actions and not doing other actions. Affordances exist where the characteristics of the object and the characteristics of the person match in a particular way. For instance, most chairs will afford sitting to most adults, but will not afford sitting to a 6-months baby, and might afford standing to someone making a speech. This concept is also applicable to other animal species, like for example a tree can afford nourishment to a giraffe but for a bird it can afford nesting. An affordance is a combination of the physical characteristics of the object and the animal, the knowledge about the object, and the needs to the animal at a particular time. In some cases, the action possibility may be harmful, in which case the animal may choose not to perform the action. For example a knife affords cutting into various surfaces 
because it has a blade. If someone grasps it by the handle it affords cutting into bread or cut through paper but it also affords injury if grasped by the blade. Another example is about apertures. An aperture will only afford passage if it is wider than the individual. If it is narrower it may afford passage if the individual rotates upon himself [18]. Affordances are based on experience, in the sense that people learn to perceive the relevant characteristics of the environment and objects within it. This means they will be common to many individuals (e.g., passing through apertures which are large enough) but different from one individual to another (e.g., a rugby player, a gymnast, or a child will fit through different apertures). The link between perception and action which guides people's decision evolves over time through experience [19].

After the initial study by Warren and Whang [18] testing affordances, other studies have followed which explore and test the notion of affordances, such as [20, 21]. One crucial finding was that the possibilities for action available to an individual are scaled to the individual's body. This scaling factor is important because it links object properties and individual's dimensions through an invariant value; this means there is a lawful relation underpinning (at least some) affordances. Such lawful relations have been found in various animals. In human participants, this was found in stair climbing where participants deem a stair climbable (without the aid of hands) if the raiser is smaller than 0.88 their leg length [22]. This was also found to be the case in passing through apertures where participants rotate their shoulders over their longitudinal axis if the aperture is smaller than 1.4 the width of their shoulders [18].

\section{Questioning and Prototype Solution}

\subsection{Problematic}

The main purpose of this article is to evaluate the effect of an avatar on the feelings of telepresence. Avatars are very common in virtual environments. They are the alteregos of users in the virtual world. The avatar is usually seen from a first or third person perspective. Several aspects can be studied regarding the avatar, such as its dynamics, morphology or physical appearance. In addition, aspects such as the camera perspective or a visuo-motor feedback from hardware might change the ownership feelings about the avatar. The physical user interface is an important parameter to take into account because the control the user has on the avatar influences his/her behavior [23]. All of these features may influence the way a person feels towards the avatar [24]. Here, the avatar's morphology and dynamics were studied when a human user controlled it through a NUI.

The morphology of the avatar is an important feature in achieving body ownership. For instance, Tsakiris and Haggard [25] carried out the rubber hand illusion experiment with a fake hand and with a wooden stick. Their results showed that, with the rubber hand, ownership was easier to achieve than with the wooden stick. Because feelings of ownership happened with the hand, a similar result is expected if applied on a larger scale to a scaled-to-user avatar. This was done by Petkova and Ehrsson [10] in a study using a camera on a mannequin in the real world. 
The dynamics of the avatar may also help to induce the feelings of telepresence and ownership on its user. If there is real-time congruence between the movements of the user and the movements of the avatar, then the feelings of telepresence and ownership should be greater than with incongruent movements. This is supported by the experiment carried out by Kalckert and Ehrsson [26]. These authors showed that the rubber hand illusion can be induced through a simple visuomotor correlation, without the need for a tactile stimulation as had been used in the original study of Botvinik and Cohen [6].

The study presented here is composed with two experimental conditions. In one of the conditions, the avatars are morphologically proportional (similar) to each participant. It is possible to have a dynamic avatar fully proportional to the user thanks to a full body motion capture. In the other condition, the avatar resembles the first one in how it looks, but it is the same (standard) for every participant. In addition, the movements of this standard avatar do not exactly match the participant's movements, as it only moves sideways and rotates upon itself. Aside from the avatar conditions, there was also a speed condition: fast and slow.

\subsection{Developed Tool}

The ATTAVE (Avatar Telepresence Testing: Affordances in Virtual Environments) is the name of the prototype developed. The ATTAVE is a virtual environment where the avatars exist and where experiments using affordances are performed. This virtual environment will be the same for both avatar conditions. Thus, the only parameters that change in the experiment are the avatar's characteristics. The design of the prototype is based on a study performed by Warren and Whang [18]. The authors evaluated how people passed through apertures considering the shoulder width of the participant and the ratio between the aperture and their shoulder width. The participants passed through several apertures of various sizes and the degree of their shoulder rotation was recorded. There were two speed conditions: a slow and a fast walking speed. Results showed that the participants only walked frontally through the aperture when the ratio between the aperture and the shoulders was smaller than 1.4. The present study will be similar to the one performed by Warren and Whang [18], but will be performed in a virtual environment. By replicating a study performed in the real world, telepresence can be verified if the same behaviors that happen in the real world also happen in the virtual environment.

The display of ATTAVE consists of a virtual scenario showing a long treadmill moving towards a visible avatar (and also towards the participant). The avatar resembles a wooden mannequin and is visible from head to knees, as the viewpoint of the participant is $2 \mathrm{~m}$ behind the avatar. The treadmill is enclosed on the side by tall walls. On the treadmill itself there are frontal green walls with an aperture on the left, centre or right side of the wall. All surfaces have texture as can be seen in Figure 1. The participants could control the translation and rotation of the avatars by physically moving side to side and rotating their own shoulders. Shoulder rotation proportionally slowed down the treadmill. The participants' task was to avoid collisions and pass through all doors as fast as possible. 


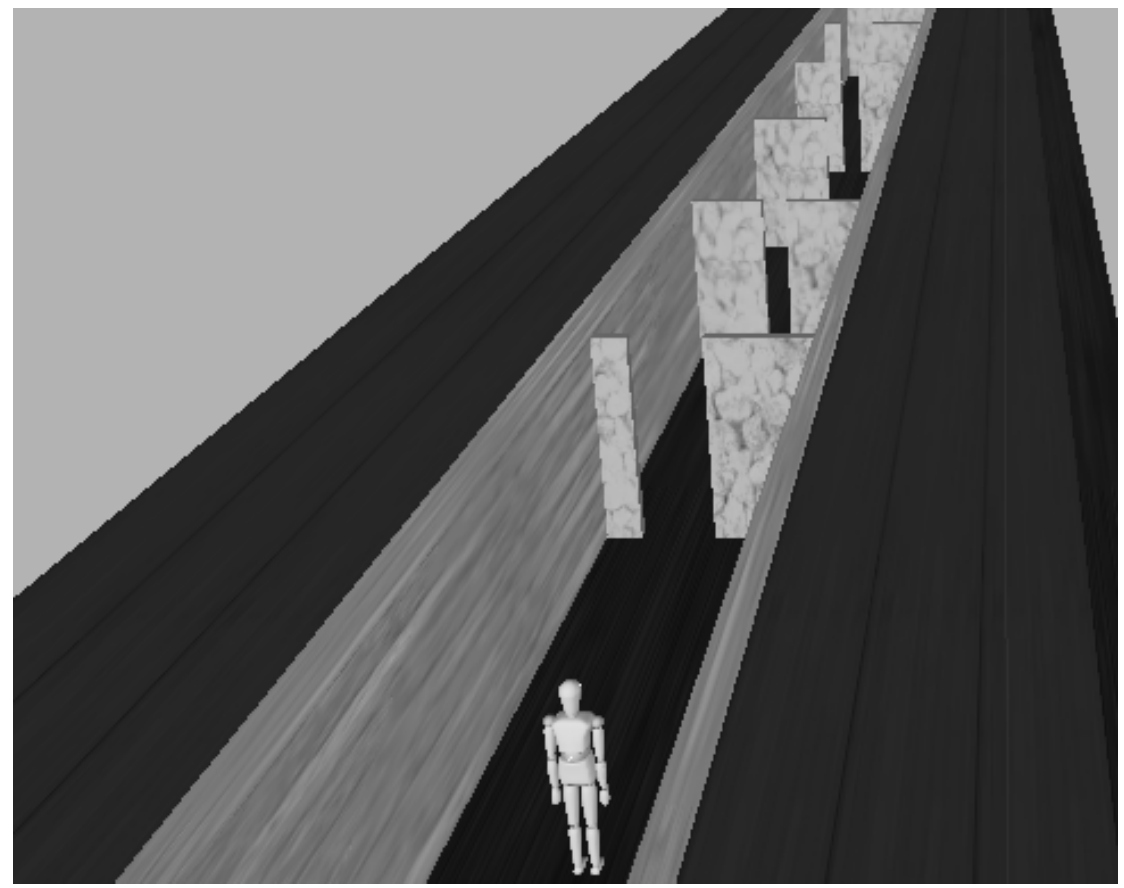

Fig. 1. ATTAVE seen from above (this was not the perspective used in the study)

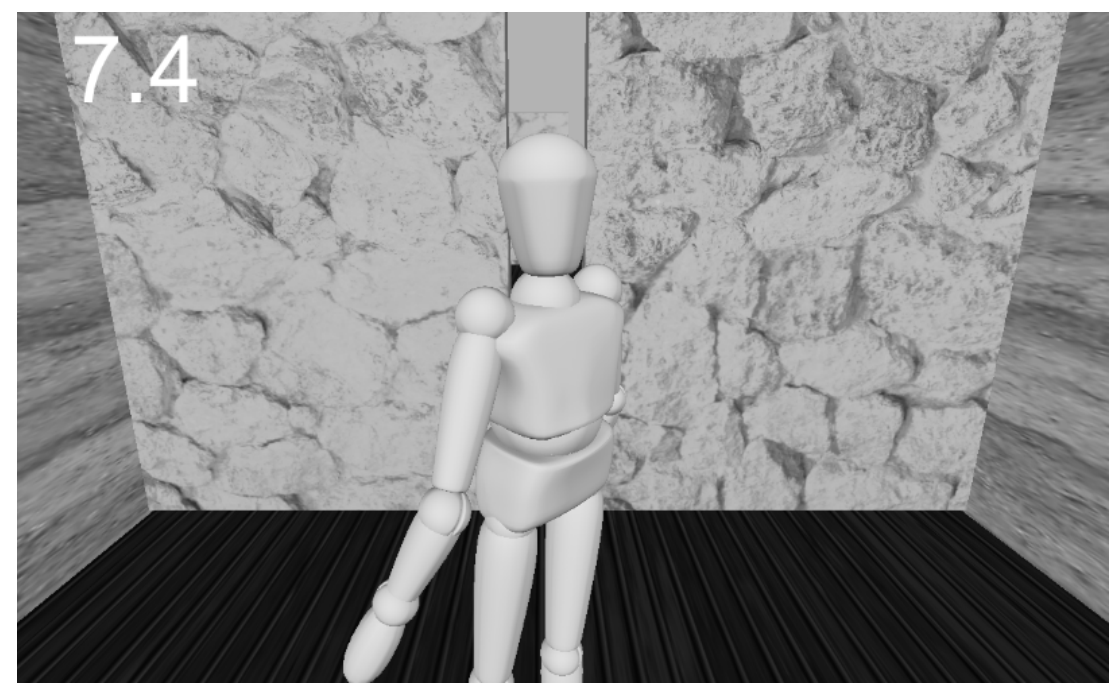

Fig. 2. Virtual avatar and environment viewed from the participants' perspective

The software chosen for modeling the avatars was Blender. The final models resembled a wooden mannequin (Figure 2). The software used to create the animation of the (standard) avatar was iPi Recorder and iPi Mocap Studio (iPi Soft). This tool was 
chosen because it can record the movements captured by the Kinect and associate those movements to the avatar. With this method, an animation was created of a sidestep with a human model performing the action. This was the standard side-step used by the avatar in the standard condition.

Unity $3 \mathrm{~d}$ was the game engine chosen for designing ATTAVE and running the application. The advantage of Unity $3 \mathrm{~d}$ is its MonoDevelop IDE (Integrated Development Environment) integrated in the software, which facilitates the scripting part of the game. The scripting was made entirely in Java scripting language for Unity. Another reason that led to the use of Unity $3 \mathrm{~d}$ in the project was its easy integration with the Kinect, which is made possible thanks to a framework called OpenNI (Open Source Natural Interaction) provided by Primesense.

For the full body motion capture a Kinect NUI was used. This device uses an optic technology that allows detection of the human body thanks to an infrared depth camera. This choice was due to its ease to set up, as it can be ready to use in less than five minutes, and also due to its low cost compared to other equipment of the same category. Another advantage in using this system is the fact that the user does not have to wear a specific suit and, consequently, he/she has full range of movement. In order to provide the participant with audio-visual feedback, a noise and a flash next to the avatar's shoulders were displayed whenever there was a collision with a wall.

When the participant rotated the shoulders, the speed of the treadmill decreased in proportion to the cosine of the angle between the shoulders' axis and the avatar's translation axis, as described by the formula below.

$$
\text { current speed }=\text { set speed } *(1-0.4 * \sin (\text { angle }))
$$

In (1), the value of the angle is taken as 0 if the individual is in a frontal position towards the door and $90^{\circ}$ if the individual is facing the side walls. The angles are in absolute values from $0^{\circ}$ to $90^{\circ}$. The speed decrease with rotation was introduced because it simulates a normal behavior observed in the real world. People usually reduce their locomotion velocity when they have to pass through a narrow aperture. The decreasing value of 0.4 , used to calculate the current speed, was based on pilot trials. Moreover, the correlation between rotation and speed was an incentive for participants not to rotate their shoulders unless there was a danger of collision, as the rotation slowed down the treadmill and added to their total time on the task.

\section{Experiment}

\subsection{Participants}

Participants were 24 university students (18 male and 6 female, aged between 20 and 28 ), with normal or corrected-to-normal vision and varied experience in playing video games. Half of the participants performed in two conditions (similar fast, similar slow) and the other half performed in two other conditions (standard fast, standard slow). This was done to enable the study of eventual learning effects under each speed 
condition. The experiments were approved by the local ethics committee of the Nova University Lisbon.

\subsection{Setup}

The experiment was conducted in a $3 \times 3 \mathrm{~m}$ area. Participants stood $3 \mathrm{~m}$ away from a $75 \mathrm{~cm}$ height table. On the table was mounted an off-the-shelf Kinect sensor (Microsoft, for Xbox 360) and an 18" computer screen $(1440 \times 900$ pixel resolution), both connected to a PC. Three small marks on the floor indicated the positions aligned with the three apertures on the display (see Figure 3).

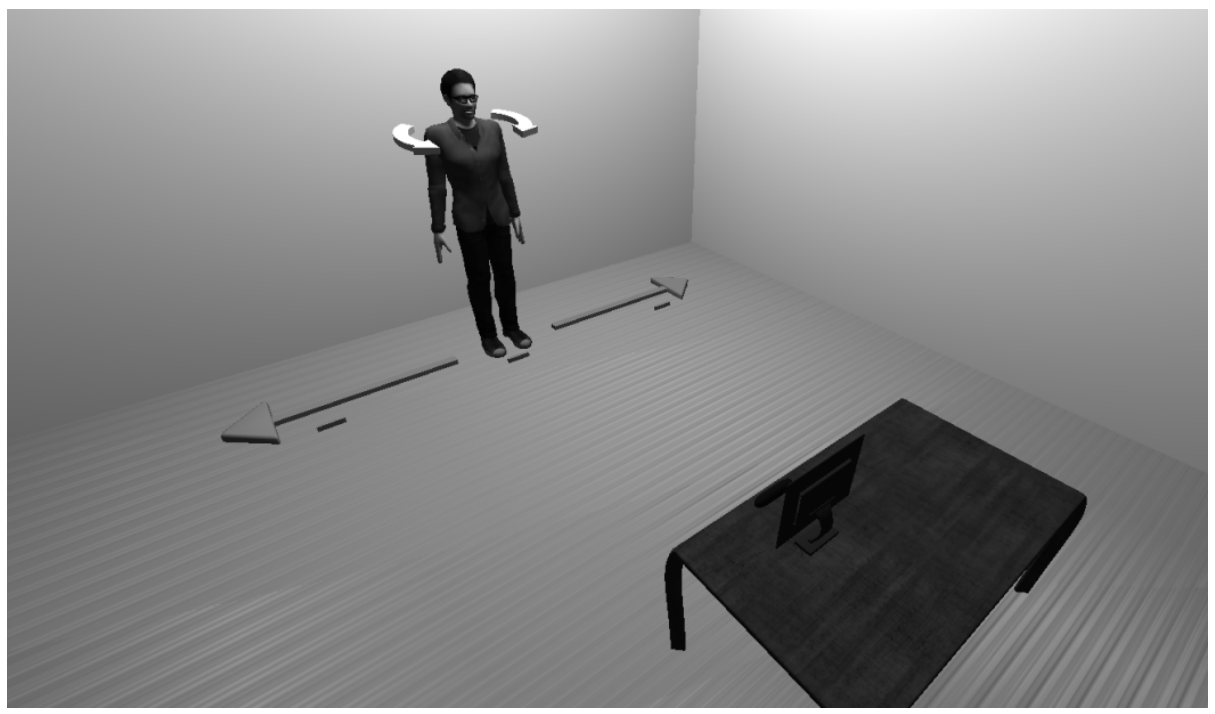

Fig. 3. Physical setup of the experiment

\subsection{Experimental Design}

Participants performed 32 trials for each of 2 speed conditions and for each of 4 sessions. Also, there were 2 avatar conditions; each used in a group of participants. The 32 trials consisted of apertures that showed in the central position with widths gradually increasing relative to the avatar's shoulders from 0.7 to 2.2 and then gradually decreasing from 2.2 to 0.7 (in steps of 0.1 ). When the avatar passed through each of these apertures, the value corresponding to the angle between the shoulders was recorded. These trials were alternated with 32 dummy trials with apertures of constant size shown in the right and left side positions. These side apertures remained twice the shoulder width of the avatar and were not used for data collection. Every aperture was 10 meters away from the next aperture. The two speed conditions were slow and fast (respectively 5 and $10 \mathrm{Km} / \mathrm{h}$ ). They were taken from the walking speeds reported by Warren and Whang [18] and adjusted during pilot testing. The avatar condition consisted of manipulating the morphology and movements of the avatar. In the similar 
avatar condition the avatar was anatomically proportional to the dimensions of the participant and all segments were animated to mimic the natural movements of the participant. In the standard condition the dimensions of the avatar were standard for all participants and the avatar had only two degrees of freedom: translation sideways and rotation upon itself (see arrows on Figure 3). An animation of a sidestep was implemented on the avatar when the participant performed a sidestep. This animation was recorded with a natural user interface and results from a sidestep performed by a human being.

The ratio between each virtual door and the avatar's shoulders width was the independent variable manipulated. The dependent variable was the angle between the shoulders during the passage through each aperture.

\subsection{Procedure}

The experiment started with participants reading and signing the consent form. Then, the Kinect was calibrated to the participants' movements. Participants were instructed to avoid collisions and complete the task in the shortest possible time, and were informed that shoulder rotation proportionally decreased the speed of the treadmill. In each session, participants completed the increasing-decreasing series in the slow condition followed by the fast condition. Participants were asked to fill in a questionnaire adapted from Witmer et al. [27]. Finally, the measure of participants' height and shoulder width was taken. In total, each session lasted about 20 minutes.

\subsection{Results}

Data Analysis. The main dependent variable was the critical ratio after which the participant passed without rotation. Following Warren and Whang [18], the two values of the critical ratio of shoulder rotation from the increasing-decreasing series were averaged. The critical ratio was that with an angle smaller than $16^{\circ}$ and after which all angles were smaller than $16^{\circ}$ (one exception was permitted provided the angle was smaller than $40^{\circ}$ and the average angles remained smaller than $16^{\circ}$ ). A critical ratio was calculated for each participant, condition, and session and these were used in the data analysis.

To examine learning effects from session to session, the critical ratios were submitted to a multivariate repeated measures analysis of variance (MANOVA) with the factor session (4 levels), and using the 4 conditions as measures (slow-similar, fastsimilar, slow-standard, fast-standard). Based on the results of this analysis, the averages of the last 3 sessions were used in the remaining analysis.

To examine the effect of conditions on the critical ratios, the individual critical ratios from the last 3 sessions were averaged and submitted to a repeated measures analysis of variance (ANOVA) with factors speed (2 levels: slow and fast) and avatar (2 levels: similar and standard). The same analysis was conducted for the total durations, that is, the time that the participants took to complete the task by going through all the apertures. The same analysis was also used to test the effects on collisions. 
To examine how participants felt regarding the experienced environment, the scores for each dimension of the questionnaires were averaged and submitted to a multivariate repeated measures analysis of variance (MANOVA) with factors avatar (2 levels: similar and standard) and session (4 levels) and using the 5 dimensions as measures (realism, possibility, quality, ownership, and self-evaluation).

Finally, Pearson's $r$ was used to test the correlation between critical ratios and the dimension of ownership as measured by the questionnaire.

Learning of Critical Ratios. Overall, there was a significant learning effect on the critical ratios, $\mathrm{F}(12,86)=6.85, \mathrm{p}<.001, \eta^{2}=.49$. This was reflected in the four conditions: slow-similar, $\mathrm{F}(3,33)=17.74, \mathrm{p}<.001, \eta^{2}=.62$; fast-similar, $\mathrm{F}(3,33)=$ $3.08, \mathrm{p}<.05, \eta^{2}=.22$; slow-standard, $\mathrm{F}(3,33)=8.74, \mathrm{p}<.001, \eta^{2}=.44$; and faststandard, $\mathrm{F}(3,33)=3.07, \mathrm{p}=.05, \eta^{2}=.22$.

Pairwise comparisons showed significant differences between the first and the last three sessions which did not differ between them (see Figure 4).

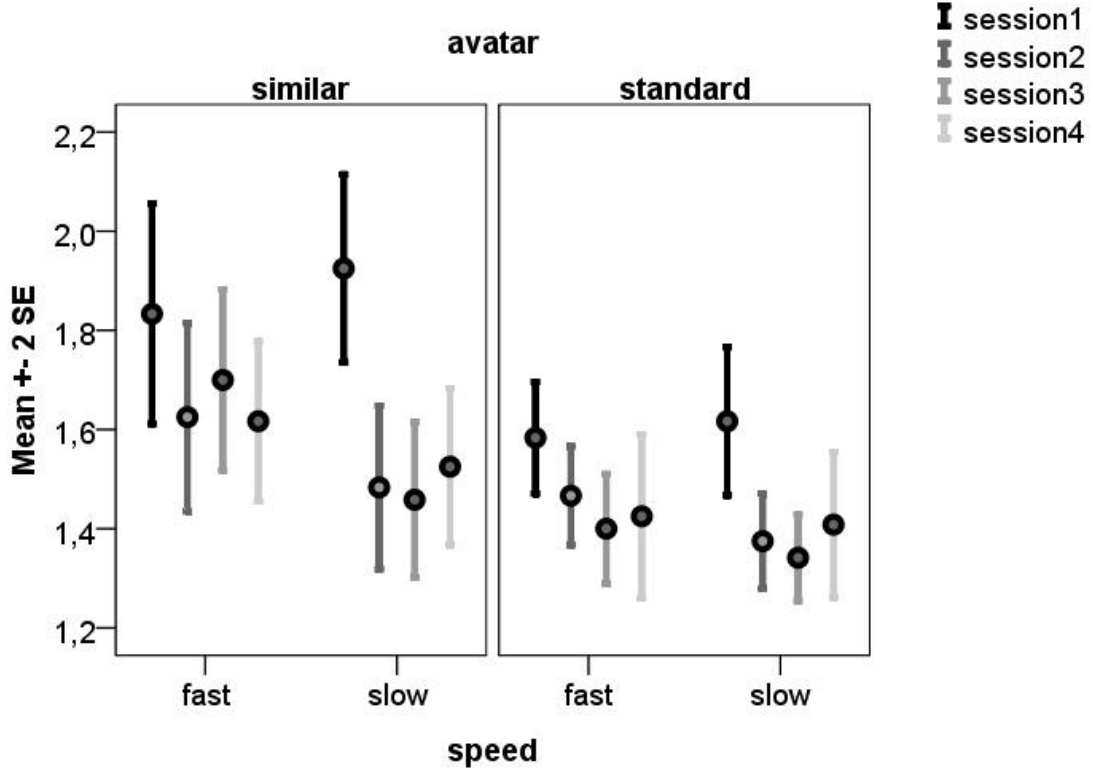

Fig. 4. Average critical ratios for the four conditions over the four sessions

Critical Ratios. There was a strong tendency for an effect of critical ratio on avatar, $\mathrm{F}(1,11)=4.62, \mathrm{p}=.055, \eta^{2}=.30$ (Figure 5 ), which was caused by participants rotating their shoulders at smaller critical ratios when the avatar was standard than when the avatar was similar ( standard $\mathrm{M}=1.40$, se $=0.05$, similar $\mathrm{M}=1.57$, $\mathrm{se}=0.07$ ). 


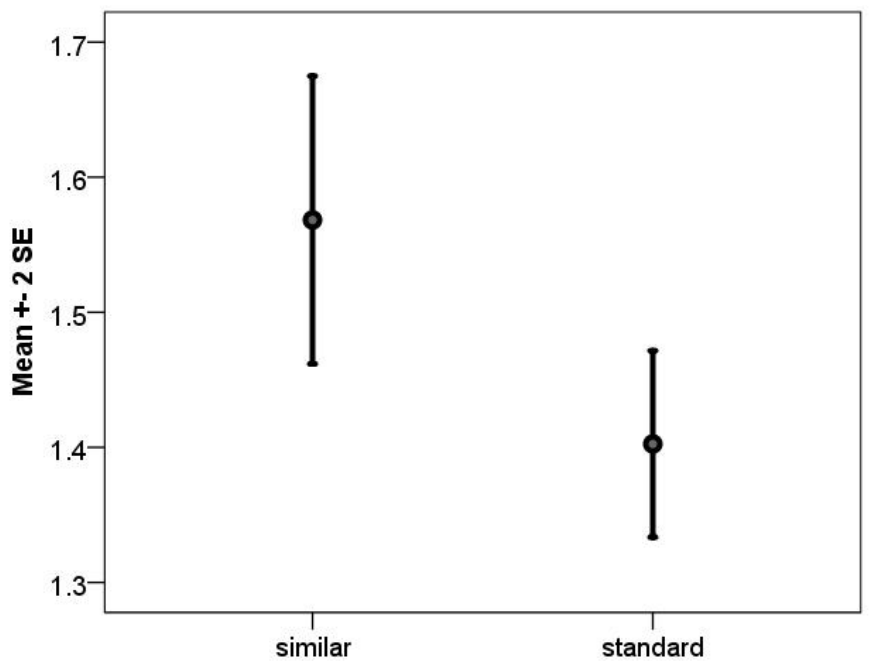

Fig. 5. Effect of the avatar on the critical ratios

There was a significant main effect of critical ratio on speed, $\mathrm{F}(1,11)=5.13$, $\mathrm{p}<.05$, $\eta^{2}=.57$ (Figure 6 ). This was caused by participants rotating their shoulders at smaller critical ratios in the slow condition compared to the fast condition (slow $\mathrm{M}=1.43$, se $=0.04$; fast $\mathrm{M}=1.54$, $\mathrm{se}=0.05$ ).

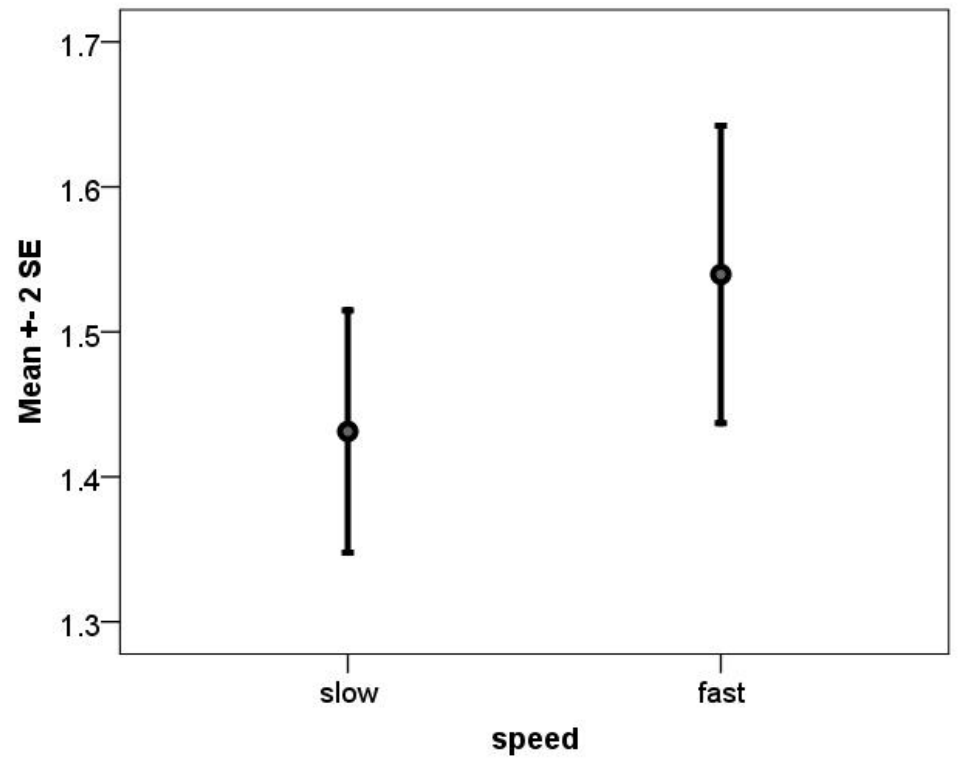

Fig. 6. Effect of speed on critical ratios 
There was no Speed $\times$ Avatar interaction, $F(1,11)=2.51$, however it is noteworthy that the effect of speed was more marked when avatars were similar than when avatars were standard (Figure 7).

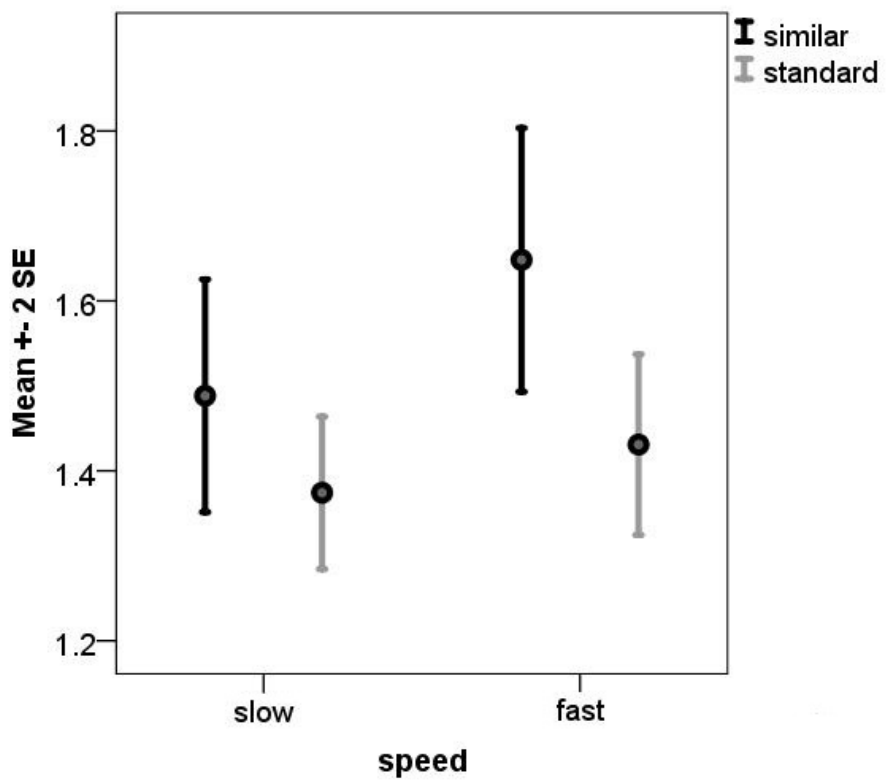

Fig. 7. Effect of speed condition and avatar on the critical ratios in the four sessions (black bars represent the similar avatar and grey lines represent the standard avatar)

Duration. The main effect of duration on speed, $\mathrm{F}(1,11)=7.07, \mathrm{p}<.001, \eta^{2}=.99$, was caused by the condition itself ( 2.2 ). The main effect of avatar was not statistically significant, $F(1,11)=1.93$, although participants took slightly shorter in the standard than in the similar condition (standard $\mathrm{M}=259.3$, $\mathrm{se}=4.06$, similar $\mathrm{M}=365.6$, $\mathrm{se}=1.6$ ). There was a significant Speed $\times$ Avatar interaction, $\mathrm{F}(1,11)=19.09, \mathrm{p}<.001, \eta^{2}=.63$. This was because in the slow condition, participants performed slower with similar avatars (slow standard $\mathrm{M}=468.3$, se $=5.03$, slow similar $\mathrm{M}=484.7$, se $=2.05$ ), whereas in the fast condition participants performed faster with similar avatars (fast standard $\mathrm{M}=250.2$, $\mathrm{se}=$ 4.42, fast similar $\mathrm{M}=246.4$, $\mathrm{se}=1.32$ ).

Collisions. There were no significant main effects of collisions on speed, $F(1,11)=$ 0.0 , avatar, $F(1,11)=2.19$, and no significant interaction effect, $F(1,11)=0.40$. On average there were 2 collisions on each session across conditions.

Questionnaire. Overall, there was no significant main effect of avatar, $F(2,7)=1.83$ or session, $F(2,7)=0.93$. However, there was a significant Avatar $\times$ Session interaction, $F(15,80)=1.87, \mathrm{p}<.05, \eta^{2}=.24$. This significant interaction was reflected in 
three dimensions: realism, $\mathrm{F}(3,33)=4.00, \mathrm{p}<.05, \eta^{2}=.27$; ownership, $\mathrm{F}(3,33)=$ $3.93, \mathrm{p}<.05, \eta^{2}=.26$ and self-evaluation, $\mathrm{F}(3,33)=3.17, \mathrm{p}<.05, \eta^{2}=.22$. This interaction occurred because feelings of realism, ownership and self-evaluation increased in the similar condition and decreased in the standard condition (Figure 8).

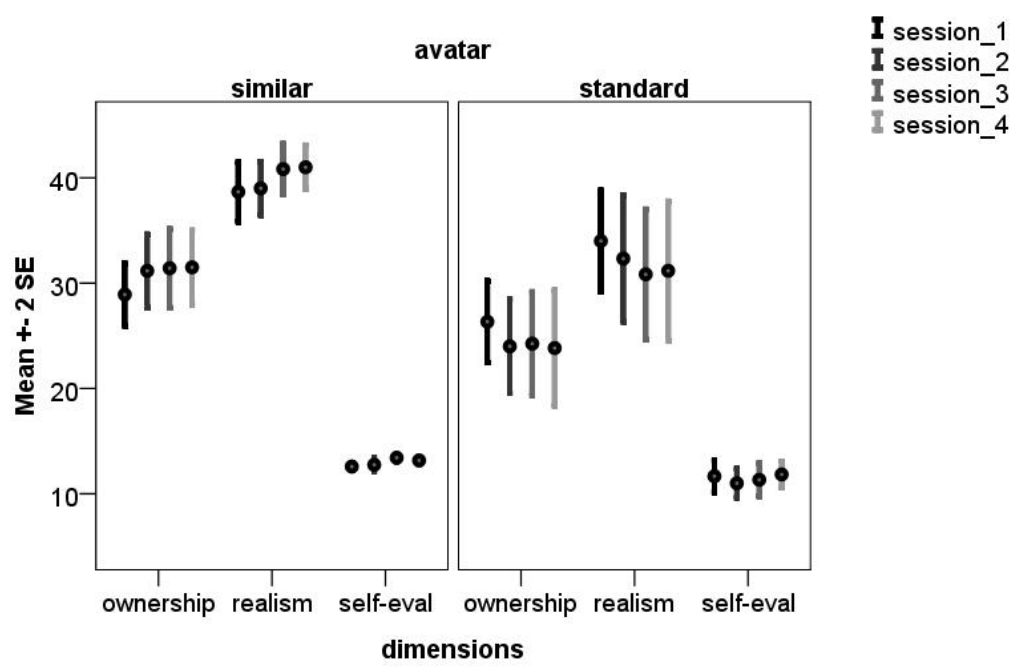

Fig. 8. Questionnaires results for the three dimensions over sessions

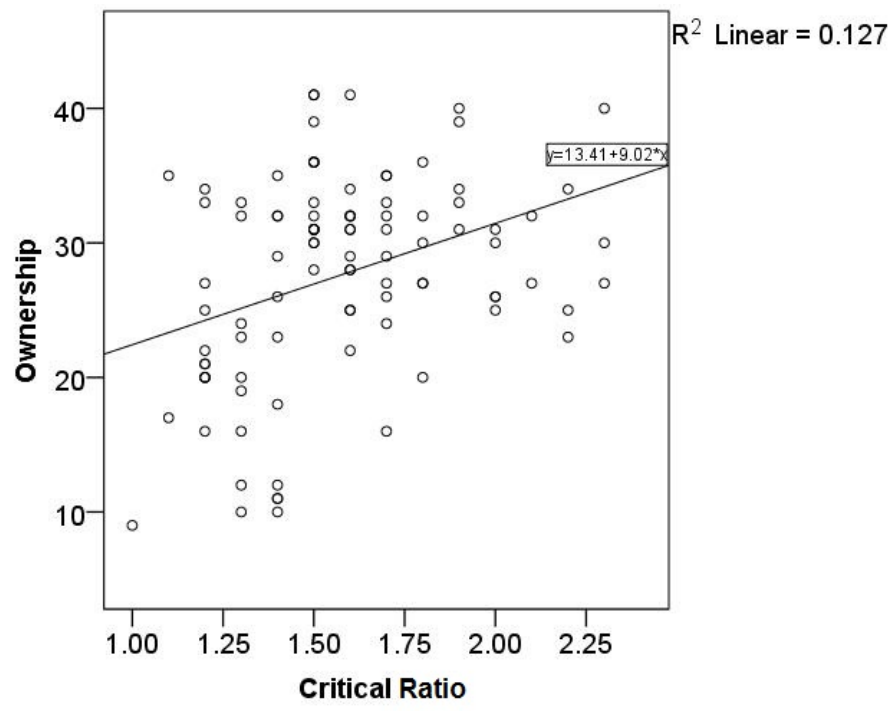

Fig. 9. Scatter plot and Pearson's r 
Ownership and Critical Ratios. Overall, there was a small, positive correlation between feelings of ownership and critical ratios, $\mathrm{r}=0.36, \mathrm{n}=96, \mathrm{p}<0.05$ indicating that increases in one variable were accompanied by increases in the other variable (Figure 9).

\section{Discussion}

The main objective of this research was to study whether the dynamics and the morphology of an avatar would reflect on the feelings of telepresence and ownership of the participant. Also, to know whether affordances were used in a virtual environment the same way they are in a real environment. In order to perform the experiment, a prototype named ATTAVE was developed. ATTAVE possess a virtual environment where the participant's avatar performs the experimental task. The user controlled the avatar through a natural motion capture carried out by a Kinect NUI. There were two avatar conditions to test: one morphologically proportional to the user and that replicated his/her movements; and another with an avatar that was identical for every participant, and exhibited limited mobility, as it could only rotate upon himself and step sideways.

The results show that participants adjusted to the virtual environment after taking their first session. This learning effect is only significant in the first session, and the other three sessions were similar to each other in a same condition. The learning effect that occurred in the first session indicates that participants learned to use appropriate information, provided by the virtual environment, to solve the problem of passing through apertures. It is usual in Human-Machine Interaction (HMI) that users take time to adapt to the artifact. For instance, Peters et al. [28] showed that six trials on a teleoperated robotic arm are necessary to have an accurate representation of the task. On the contrary to a simple tool held in hand, the remote or virtual interaction is not straightforward and involves a specific design of the artifact to be ergonomically adapted to the human user [29]. After learning, the critical ratios obtained in the virtual environment (1.4 and 1.57) were very similar to those in the real environment (1.4 reported by Warren and Whang [18]), which demonstrates that people perceive similar body-scaled affordances in the virtual environment as in real environments.

The effect of the different avatars was significant. Participants with the standard avatar had a smaller critical ratio than the participants with the similar avatar (1.4 vs. 1.57). It means that participants who controlled a similar avatar tended to keep a greater safety margin between the body and wall, compared with a standard avatar. This result can suggest a higher feeling of agency for the similar avatar by the individual, who may care about his/her alter-ego and therefore make sure it does not collide with the wall. In contrast, participant seemed less concerned regarding the standard avatar. This interpretation is supported by the analysis of the correlation between the ownership questionnaire and the critical ratios. There was a positive correlation between these two variables, albeit small, which suggests that the rise in the critical ratios is related with an increase in the feelings of ownership. 
The higher critical ratios in the similar condition compared to the standard condition could also be explained by distraction because the similar condition involved seeing more of their own movements in the avatar's movements. The amount movements may have led the participants to attend to something other than the shoulders and the apertures. The movements of the standard avatar were much more restricted, which decreased the amount of cues available. In the standard condition, the participant's body controlled the avatar as if it were a simple joystick. Consequently, this condition can be interpreted in terms of a classical remote control of a teleoperated machine. In a study carried out by Moore et al. [30], the user controlled a robot and supervises the environment by means of a camera on top of the robot. The operator's task was to judge whether or not the robot could pass through apertures of various sizes. The results indicate that the participants judged the robot could pass even when it could not. Authors argued that the results might be influenced by structural and morphological aspects. The same effect could explain the results of the experiment presented here. The fact that the individual underestimates the aperture width could signify that he/she considers the standard avatar as a machine-like character and, consequently, he/she can hardly be engaged in an ownership process with it.

In addition, one aspect that can increase the immersiveness in a virtual environment is the type of interaction between human and machine. If the interaction is done through a classic controller such as a mouse or joystick, the users need to learn the mapping between their own movement and its consequence in the virtual world $[31,32]$. On the contrary, the mapping is facilitated if the interaction is done through a full motion capture. Research has shown that natural user interfaces, in which users can recognize their own movements in the virtual environment, are more immersive $[33,34]$. It could explain why the similar avatar condition seems to bring a higher feeling of ownership compared with the standard condition.

Analyzing the effect of speed on the critical ratios, it is observable that in both avatar conditions the critical ratio is larger when the speed is higher. This happens because at higher speeds people leave larger safety margins. In the real world, when an individual is confronted with an aperture, he/she will reduce his/her speed in order to fit through without colliding. In this project, the only way to decrease the speed was by rotating the shoulders, which resulted in a higher critical ratio. The relation between speed and accuracy is well-known in the area of motor control and was described for the first time in Fitts' law [35]. The fact that the participants of this experiment reproduced this natural motor control adaptation suggests their immersion into the virtual environment (ATTAVE).

Finally, the questionnaire showed that the similar avatar elicited an increasing feeling of ownership and realism over the four sessions. In contrast, the standard avatar caused a decreasing feeling of ownership and realism from session to session. This result means that an agency process in which the participants consider the similar avatar as a natural extension of themselves seems to happen and to increase through the interaction with the avatar. In contrast, with the standard avatar, participants may have become bored due to the avatar's movement not being as diverse as their own. This lack of biological motion could lead the participants to act as if they were controlling a machine instead of a virtual representation of themselves. Overall, 
considering the questionnaire results and the affordances described through the critical ratios, it seems that an avatar with natural movements and tailored the morphology of the user can significantly enhance the feelings of body ownership.

\section{References}

1. Minsky, M.: Telepresence. Omni 2, 45-51 (1980)

2. Slater, M., Usoh, M., Steed, A.: Depth of presence in virtual environments. Presence 3, 130-144 (1994)

3. Rybarczyk, Y., Mestre, D.: Effect of visuo-manual configuration on a telerobot integration into the body schema. Le Travail Humain 76, 181-204 (2013)

4. Rybarczyk, Y., Hoppenot, P., Colle, E., Mestre, D.: Sensori-motor appropriation of an artefact: A neuroscientific approach. In: Inaki, M. (ed.) Human Machine Interaction - Getting Closer, pp. 187-212. InTech, Rijeka (2012)

5. Sumioka, H., Nishio, S., Ishiguro, H.: Teleoperated android for mediated communication: Body ownership, personality distortion, and minimal human design. Social Robotic Telepresence 32 (2012)

6. Botvinick, M., Cohen, J.: Rubber hands 'feel' touch that eyes see. Nature 391, 756-756 (1998)

7. Yuan, Y., Steed, A.: Is the rubber hand illusion induced by immersive virtual reality? In: Virtual Reality Conference, pp. 95-102. IEEE Press (2010)

8. Tsakiris, M., Prabhu, G., Haggard, P.: Having a body versus moving your body: How agency structures body-ownership. Consciousness and Cognition 15, 423-432 (2006)

9. Ehrsson, H.H., Spence, C., Passingham, R.E.: That's my hand! Activity in premotor cortex reflects feeling of ownership of a limb. Science 305, 875-877 (2004)

10. Petkova, V.I., Ehrsson, H.H.: If I were you: Perceptual illusion of body swapping. PloS One 3, e3832 (2008)

11. Maravita, A., Iriki, A.: Tools for the body (schema). Trends in Cognitive Sciences 8, 79-86 (2004)

12. Iriki, A., Tanaka, M., Iwamura, Y.: Coding of modified body schema during tool use by macaque postcentral neurons. Neuroreport 7, 2325-2330 (1996)

13. Maselli, A., Slater, M.: The building blocks of the full body ownership illusion. Frontiers in Human Neuroscience 7, 83 (2013)

14. Armel, K.C., Ramachandran, V.S.: Projecting sensations to external objects: Evidence from skin conductance response. Proceedings of the Royal Society of London. Series B: Biological Sciences 270, 1499-1506 (2003)

15. Slater, M., Perez-Marcos, D., Ehrsson, H.H., Sanchez-Vives, M.V.: Inducing illusory ownership of a virtual body. Frontiers in Neuroscience 3, 214 (2009)

16. González-Franco, M., Peck, T.C., Slater, M.: Virtual Embodiment Elicits a Mu Rhythm ERD When the Virtual Hand is Threatened. In: 8th International Brain Research Organisation, Congress of Neuroscience (2011)

17. Gibson, J.J.: The ecological approach to visual perception. Houghton Mifflin, Boston (1979)

18. Warren, W.H., Whang, S.: Visual guidance of walking through apertures: Body-scaled information for affordances. Journal of Experimental Psychology: Human Perception and Performance 13, 371-383 (1987) 
19. De Oliveira, R.F., Damisch, L., Hossner, E.J., Oudejans, R.D., Raab, M., Volz, K.G., Williams, A.M.: The bidirectional links between decision making, perception, and action. Progress in Brain Research 174, 85-93 (2009)

20. Mark, L.S.: Eyeheight-scaled information about affordances: A study of sitting and stair climbing. Journal of Experimental Psychology: Human Perception and Performance 13, 361-370 (1987)

21. Esteves, P.T., De Oliveira, R.F., Araújo, D.: Posture-related affordances guide attack in basketball. Psychology of Sport and Exercise 12, 639-644 (2011)

22. Warren, W.H.: Perceiving affordances: Visual guidance of stair climbing. Journal of Experimental Psychology: Human Perception and Performance 10, 683-703 (1984)

23. Meadows, M.S.: I, avatar. New Riders Press, Berkley (2008)

24. Castronova, E.: Theory of the Avatar. Cesifo (2003)

25. Tsakiris, M., Haggard, P.: The rubber hand illusion revisited: Visuotactile integration and self-attribution. Journal of Experimental Psychology: Human Perception and Performance 31, 80-91 (2005)

26. Kalckert, A., Ehrsson, H.H.: Moving a rubber hand that feels like your own: A dissociation of ownership and agency. Frontiers in Human Neuroscience 6, 40 (2012)

27. Witmer, B.J., Jerome, C.J., Singer, M.J.: The factor structure of the Presence questionnaire. Presence 14, 298-312 (2005)

28. Peters, R.A., Campbell, C.L., Bluethmann, W.J., Huber, E.: Robonaut task learning through teleoperation. In: IEEE International Conference on Robotics and Automation, pp. 2806-2811. IEEE Press (2003)

29. Rybarczyk, Y., Mestre, D.: Effect of temporal organization of the visuo-locomotor coupling on the predictive steering. Frontiers in Psychology 3, 239 (2012)

30. Moore, K.S., Gomer, J.A., Pagano, C.C., Moore, D.D.: Perception of robot passability with direct line of sight and teleoperation. Human Factors: The Journal of the Human Factors and Ergonomics Society 51, 557-570 (2009)

31. De Oliveira, R.F., Wann, J.P.: Driving skills of young adults with developmental coordination disorder: Regulating speed and coping with distraction. Research in Developmental Disabilities 32, 1301-1308 (2011)

32. Wise, S.P., Murray, E.A.: Arbitrary associations between antecedents and actions. Trends in Neurosciences 23, 271-276 (2000)

33. Bruder, G., Steinicke, F., Hinrichs, K.H.: Arch-explore: A natural user interface for immersive architectural walkthroughs. In: IEEE Symposium on 3D User Interfaces, pp. 75-82. IEEE Press (2009)

34. Francese, R., Passero, I., Tortora, G.: Wiimote and Kinect: Gestural user interfaces add a natural third dimension to HCI. In: Proceedings of the International Working Conference on Advanced Visual Interfaces, pp. 116-123. ACM Press (2012)

35. Fitts, P.M.: The information capacity of the human motor system in controlling the amplitude of movement. Journal of Experimental Psychology 47, 381-391 (1954) 